\title{
ORIGIN OF COMETS
}

"T

HE Distribution of the Directions of Perihelia of Long-Period Comets" is the title of a paper by J. G. Tyror in Mon. Not. Roy. Astro. Soc. (117, 4 ; 1957), in which he deals specially with Lyttleton's theory of the origin of comets (see ibid., 103, 465; 1948 ; and "The Comets and their Origin", Cambridge, 1953). This theory envisaged a process of accretion of dust particles by the Sun as it passed through interstellar dust clouds, and while it explained some of the observed features of comets and of their orbits, like many other theories it presented certain difficulties. If the theory is accepted, then comets must originally have come from preferred positions in the sky, and although the diffusion of the perihelion points of such a family would probably occur, it is considered that some traces of perihelion groupings, at lesst for families born during recent cloud passages, could be detected.

Mr. Tyror examines the distribution of perihelion points of long-period comets with the object of determining whether there is any evidence of such grouping. Previous investigations by W. H. Pickering and others suggested that there was some evidence of the grouping of aphelion points, but it has been considered advisable to revise these results in the light of additional information which has appeared since the completion of their work. The present survey does not include short-period comets and periodic comets because planetary perturbations would have eradicated reliable evidence of any original grouping; while it is true that all orbits would have been affected to some extent, the investigation has been carried out only for comets suffering slight perturbations. The whole subject is beset with difficulties because it is inevitable that available data must suffer to some extent from observational selection. A detailed description of the contents of the paper is quite impossible in a short note, and it will suffice to deal with the data utilized and the conclusions that have been reached.

Investigations of the positions of 448 comets with periods greater than 200 years were made, and of these 67 had calculated eccentricities greater than 1 , 263 equal to 1 , and 118 less than 1 . There is evidence that the perihelion points of long-period comets tend to be distributed non-randomly, and it is pointed out that this tendency may be due to observational selection or to a real grouping phenomenon, or even to a combination of both. The first of these has undoubtedly played a prominent part in the discovery of comets, and other vitiating factors in the anslysis are mentioned. The conclusions are summarized at the end of the paper and are briefly as follows.

The departure from randomness in the observed distribution of porihelion points of the comets considered is strongly suggested, and there is evidence indicating a preference for the perihelion points to lie near a particular great plane. In addition, the centre of the observed distribution appears to be significantly displaced towards a point on the celestial sphere. The identification of the plane with the plane of the Galaxy and of the point as that lying in a direction opposed to that of the Sun's motion cannot be considered unreasonable. These suggestions may be interpreted in the light of Lyttleton's accretion hypothesis, according to which the perihelion positions of comets should show a tendency to lie near the apex of the Sun's motion relative to the local interstellar cloud, and further, the distribution of perihelion points would have a preference for lying near the galactic plane.

\section{QUALITY IN MEAT}

TOW that the supply of meat is approsching the 1 level of demand, quality is again assuming economic importance. Definitions and tests of quality have been examined by D. M. Joubert of the Department of Animal Husbandry, University of Pretoria, who has also examined the economic consequences which follow from changed attitudes towards quality (Colonial Plant and Animal Products, 6 , No. $2 ; 1956)$.

Only a few decades ago quality in beef meant wellmarbled meat covered by a thick layer of fat. Not only did the average consumer during those times demand more fat owing to the bodily needs imposed by hard manual labour, but also excess fat wes used for other purposes, chiefly cooking. At present, cooking fats can be obtained from cheaper sources, the consumers perform less manual labour and are better protected in their homes against cold conditions, and consequently demand the minimum of fat with their beef. Since butchers are concentrating on carcasses of young animals, it is not unlikely that the optimum carcass weight will become even lower in future.

When considering quality in the case of mutton and lamb, the size factor is of primary importance. With the size of the average family decreasing all over the world and with cooking facilities less spacious than they used to be, the general demand is for small joints. Preference is given to a whole joint of small size over half a large one since the latter, because of its cut surface, does not retsin its succulence so well and leads to greater wastage through exudation. As in beef, a minimum of fat is preferred. With the rapid development of the dairy industry in recent years and with progress in the manufacture of margarine, the excess fat is no longer widely used as a substitute for butter and goes to waste.

Definition of quality in mutton is particularly complicated because of the differences in the taste of individuals in respect of flavour. Mutton produced from early-maturing sheep (as, for example, Southdown, Suffolk) raised and fattened in stalls may appear extremely attractive and, therefore, be labelled as of high quality. On the other hand, mutton produced from late-maturing sheop breeds (such as Scotch Blackface, and even possibly Merino) off extensive pastures may, by comparison, appear unattractive and may be labelled as being of low quality.

The quality of pork is determined primarily by the amount and nature of the subcutaneous back fat. The amount of fat preferred with pork by consumers in Britain at present is considerably less than a few decades ago, while in South Africa, for example, a large amount of fat has never been tolerated except 